is being steadily carried out. Human studies on the thalamus and cortex are already well advanced and the ingenious gate-theory for pain has stimulated valuable therapeutic studies. This volume, one of a major new series, is a valuable record of present ideas. As the editor rightly observes, in an active research field it is both necessary and usual to find conflicts of ideas between different expert contributors. Doubtless many of the ideas in this book will be superseded but it is a good summary of current views on the somatosensory system.

J. A. SIMPSON

CURRENT TOPICS IN NEUROPATHOLOGY Edited by

K. Jellinger. (Pp. 241; illustrated; price not stated.) Facultas: Vienna. 1973.

This paper-bound volume of 241 pages contains 37 of the papers presented at the 4th Danube Symposium on Neuropathology which was held in May 1973. Papers were presented by East and West German, Polish, Rumanian, Swiss, Hungarian, American, and Austrian neuropathologists. Three of the main themes of the symposium-developmental abnormalities, inflammatory and viral diseases, and cerebral tumours and their experimental inductionhave been published. There is much of interest in all of the papers but many of them have appeared as formal publications in the European literature. Only two of the papers in this volume are in English. The majority of the others have English summaries which are either too short to convey more than the simplest outlines to the non-German or have completely lost their meaning in inaccurate translation. As an example, the English summary of Dr Dudka's paper on 'Primary, circumscribed melanomas of the nervous system' seems to be far from what he meant in respect of the established existence of melanin containing cells in some meningiomas and Schwannomas.

Most of the illustrations of macroscopical preparations, light and electron microscopy have printed well and adequately supplement the texts of some detailed papers on human malformations, such as corpus callosum agenesis, and holoprosencephaly.

This is the kind of volume that those neuropathologists who attended the conference would probably like to have, but for others it would probably be worthwhile browsing in a library copy. D. DOYLE

AROMATIC AMINo ACIDS IN THE BRAIN Produced by the CIBA Foundation. (Pp. 408; \$18.60.) Elsevier/Excerpta Medica/North-Holland: Amsterdam. 1974.

The aromatic amino acids are of special significance to brain function. Thus tryptophan is, for example, important in control of protein synthesis and in relation to formation of the biogenic amine serotonin. Changes in concentration of the aromatic amino acid or its derivatives occur in sleep and stress. It appears that the level of brain tryptophan is a key factor in affective disorders and possibly in schizophrenia, while serotonin is implicated in the biochemistry of headache. Well-recognized are the neurological effects of inborn errors of tryptophan, phenylalanine, and tyrosine metabolism. There was, therefore, good reason for the holding and publication of a CIBA Foundation symposium in May 1973, at which the basic biochemistry of brain aromatic amino acids was examined in depth. Each of the 20 articles by acknowledged experts begins with an abstract and the text of about 10 pages is usually followed by an edited discussion. The result is an excellent book in which the high scientific standard is matched by a clear attractive format. The book begins with a review of factors controlling plasma amino acid (especially tryptophan) concentration. This is followed by an account of amino acid transport in the brain. Their metabolism to a range of biogenic amines, as well as nutritional factors regulating levels of the amino acids and their derivatives, are reviewed.

Attention is particularly focused on the role of tryptophan and 5-hydroxytryptamine. The final part of the book deals with the topical question of tryptophan concentration and control of brain protein synthesis. Amino acid imbalance may competitively reduce availability of amino acids for synthesis or, as has been suggested, cause polysomal disaggregation. Recent information is given on these points and the use of analogues of phenylalanine and tryptophan is examined.

A. N. DAVISON

CEREBELLAR CORTEX, CYTOLOGY AND ORGANIZATION

By S. L. Palay and V. C. Palay. (Pp. 348; illustrated; \$64.) Springer: Berlin. 1974.

This book has been produced over many years with loving care not only by the authors but by the publishers, with thoroughness, attention to detail, and calm assessment of knowledge obtained to date. It is not hard to predict that it will become a classic, a joy to possess for those who like medical books and essential consultation for all those working on the anatomy and physiology of the cerebellar cortex.

All the cellular constituents are taken in turn, Purkinje cells, mossy fibres, basket and stellate cells, and many more, dealt with vividly by 'a little history' and then our knowledge brought up-to-date by electron micrographs of impeccable quality, or freeze-etch replicas and camera lucida drawings. 
One cannot help but think that Ramon y Cajal would have approved of his successors.

D. G. F. HARRIMAN

GAS CHROMATOGRAPHY-MASS SPECTROMETRY IN NEURobiology By E. Costa and B. Holmstedt. (Pp. 175; illustrated; Dfl. 44.) North Holland: Amsterdam. 1973.

This is an easily digested book which deals with the elucidation and determination of a range of drugs and their metabolites, and other endogenous tissue constituents of known structure. It is possible that the link gas chromatography-mass spectrometry is not the rapid, ideal means of elucidating the structure of unknown biochemical substances that some of us fondly imagined, for seven of the 13 chapters deal with pharmacological or toxicological problems. The reason appears to be that the equipment is being used primarily as an expensive detector of extreme selectivity and sensitivity. The mass spectrometer is operating as a means to determine drugs and their metabolites of known structure or-in the case of the metabolites-nearly known structure. The power of this modern approach is emphasized by the determinations of such endogenous neurotransmitters as acetylcholine in various tissues and brain and pineal indole alkylamines. Another example of its value as a clinical tool is shown by the quantitative determination of 5-HAA and IAA in CSF of depressed patients, the finding that the tryptamine metabolite behaves similarly to 5-HIAA may be in keeping with the tryptamine theory of endogenous depression, and the labelling of the brain homovanillic acid of mice by allowing them simply to dwell for a short time in ${ }^{18} \mathrm{O}$-enriched atmospheres demonstrates that it is possible to label brain catecholamines in vivo, and thus avoid the problems of radiation. HVA is chosen as it is the main dopamine metabolite leaving the brain; thus, the actual brain component of the total homovanillic acid in the urine can be easily assessed. The demonstration that propanolol forms a glycol metabolite in mouse brain, only minutes after an injection, in a like manner to the catecholamine conversion to their respective glycols, gives an indication of the powerful though expensive equipment that is available to the pharmacologists.

\section{P. O. TOSELAND}

COMPENDIUM OF THE EPILEPSIES By N. Niedermeyer.

(Pp. 334; illustrated; \$14.75.) Thomas: Spring-

field, Ill. 1974.

This book contains a great deal of useful information about most aspects of epilepsy and will repay careful study. Although the author is a clinical electrographer he has clearly taken a great interest in the clinical aspects of his subject over a period of many years. The book is said to have been written for general practitioners and medical students as well as for specialists, but its wealth of detail does perhaps make it more suited for the latter than the former.

The first half of the book is devoted to basic principles and to clinical considerations. In the middle there is a single short chapter on such 'borderland' topics as migraine, paroxysmal abdominal pain, and attacks of vertigo, but these are dealt with only very cursorily. The remainder of the book is concerned with diagnosis and management, and includes chapters on the psychiatric aspects and neurosurgical treatment of epilepsy.

Not unnaturally, the subject matter of this book reflects the author's personal experience in the field of epilepsy and, as such, it is a particularly interesting contribution to the literature. It also contains much useful practical advice which could be applied with benefit to many patients with epilepsy by any clinician who is prepared to make use of it.

MAURICE PARSONAGE

NEUROSCIENCE: A LABORATORY MANUAL By James

E. Skinner. (Pp. 244; illustrated; price not stated.) Saunders: Philadelphia. 1971.

The title of this small book is misleading but it could be useful for research assistants without background experience and little time available to complete a project. In a very short space it gives an adequate account of electrical activity of the brain, basic electronics, recording of bioelectric potentials, and the elements of neuroanatomy and histology of the brain. Unfortunately, the anatomical section (which includes a dissection guide) is based on the cow and sheep brain but the stereotaxic data and experimental procedures refer to the rat. There are useful instructions, with line drawings, on the preparation of the rat for stereotaxic recording, including the construction of intracranial implant devices.

J. A. SIMPSON

TECHNiques IN NEURohistology H. M. Ralis, R. A.

Beesley, and Z. A. Ralis. (Pp. 162; illustrated; £3.40.) Butterworth: London. 1973.

This little book will be found useful in many laboratories concerned with morbid anatomy, both general and neuropathological. There are chapters on the anatomy of the nervous system for technicians, on tissue preparation, on general and neurohistological staining methods, as well as brief notes on additional techniques such as preparation for electron and fluorescence microscopy.

The chapter on general staining methods at first seems redundant, as these are available in larger 